\title{
Eurolntervention
}

\section{Scanning electron microscopic assessment of the biodegradable coating on expanded biolimus-eluting stents}

\author{
Mounir W.Z. Basalus, MD; K. Gert van Houwelingen'1, MD; Marc Ankone², MSc; \\ Frits H.A.F de Man'1, MD, PhD; Clemens von Birgelen ${ }^{1,2 *}$, MD, PhD \\ 1. Department of Cardiology, Thoraxcentrum Twente, Enschede, The Netherlands; 2. Institute for Biomedical Technology (BMTI), \\ University of Twente, Enschede, The Netherlands
}

The authors have no conflict of interest to declare.

While no financial support was obtained, free samples of DES were obtained from a Dutch supplier for the purpose of this scanning electron

microscopic assessment.

\section{KEYWORDS}

Biodegradable polymer coating; scanning electron microscopy; drug-eluting stent; percutaneous coronary intervention; coronary artery disease; BioMatrix

\begin{abstract}
Aims: Biodegradable coatings on drug-eluting stents (DES) may help to avoid adverse long-term effects of DES such as late/very late stent thrombosis which is partly attributed to durable polymers. As the postexpansion morphology of biodegradable coatings is greatly unknown, we investigated the polylactic acid coating on biolimus-eluting BioMatrix stents.

Methods and results: Scanning electron microscopy (SEM) was used to carefully examine five $3.5 \mathrm{~mm}$ stents following expansion at 6-14 atm (maximum 7\% overstretch). SEM examination demonstrated only mild cracks of the coating after stent expansion at $6 \mathrm{~atm}$. An increase in expansion pressure, associated with mild stent overstretch, resulted in more severe cracks. Lifting of the coating together with few sites of partial detachment of fragments was noticed after stent expansion in water at 14 atm; these irregularities further increased after aggressive oversized partial postdilatation with a $5.0 \mathrm{~mm}$ non-compliant balloon with additional secondary cracks.

Conclusions: SEM assessment suggests a relatively low elasticity of the biodegradable coating on BioMatrix stents. At nominal pressure, stents showed predominantly mild cracks of the coating, while cracks increased after slight overstretch. Aggressive overexpansion of the stent, such as sometimes required in left main bifurcation stenting, worsened cracks and led to some detachment of fragments of the coating in vitro.
\end{abstract}

\footnotetext{
* Corresponding author: Thoraxcentrum Twente, MST, Department of Cardiology, Haaksbergerstraat 55, 7513ER Enschede, The Netherlands

E-mail: c.vonbirgelen@mst.nl
} 


\section{Introduction}

Drug-eluting stents (DES) reduce the need for re-intervention after percutaneous coronary interventions, ${ }^{1-3}$ however, late stent thrombosis remains an issue which is partly attributed to inflammation possibly induced by durable polymer (in DES coatings) ${ }^{4-7}$ Biodegradable coating on DES has the promise to avoid potential adverse long-term effects of DES coating. ${ }^{8}$ The concept of a biodegradable drug-eluting coating was tested in combination with different antiproliferative drugs. ${ }^{9-11}$ One of these combinations is a biolimus-eluting polylactic acid (PLA) coating, ${ }^{12}$ which has recently been investigated in the LEADERS trial. ${ }^{13}$ The physical properties of the PLA coating on this DES may differ from that of durable polymer coatings on conventional DES. Scanning electron microscopy is an attractive modality to examine the coating morphology of DES. Only a few SEM data have been published on durable polymer-based DES, ${ }^{14-17}$ while there is no published SEMdata on biodegradable polymer-coated DES. This motivated us to examine the post-expansion morphology of the coating on BioMatrix stents using scanning electron microscopy (SEM).

\section{Materials and methods}

\section{Investigated stents}

At the University of Twente in Enschede, The Netherlands, we prospectively performed in vitro SEM examinations of five BioMatrix ${ }^{\mathrm{TM}}$ (Biosensors Europe SA, Morges, Switzerland) stents, being expanded in air or water at different balloon pressures, which resulted in stent dimensions that ranged from the nominal size (one sample) via mild overstretch (three samples) up to aggressive overstretch (one sample). These stents (sterile packed; expiration date not passed) were provided to us by the company Biosensors via its Dutch supplier for the purpose of SEM examination at the University of Twente. The BioMatrix stent is based on the stainless steel S-Stent ${ }^{\mathrm{TM}}$ (Biosensors International, Newport Beach, CA, USA) platform covered with a primer of parylene $C$ and on the abluminal side of the stent a mixture of biolimus-A9 and a PLA polymer. ${ }^{12}$ PLA coating is biodegradable, and expected to be totally degraded within approximately nine months. Of note, these stents have a subtle difference versus BioMatrix stents of the LEADERS trial; that is, paralyne $C$ was not used in priming the stents that were used in the LEADERS trial.

\section{DES expansion protocol in vitro}

All five stents were expanded under sterile conditions in the laboratories of the Institute for Biomedical Technology at the University of Twente under a laminar air flow hood. The experiments were performed by an experienced interventional cardiologist and a laboratory technician, assisted by a research fellow. The first two samples (samples 1 and 2) were expanded at 6 and 10 atm (in air at $18^{\circ} \mathrm{C}$ ), which corresponds to expected stent diameters of $3.50 \mathrm{~mm}$ (the stent diameter at nominal pressure) and $3.62 \mathrm{~mm}$ (103\% of the stent diameter at nominal pressure), respectively. Three stents (sample 3,4, and 5) were expanded at $14 \mathrm{~atm}$ (expected diameter of $3.74 \mathrm{~mm}=107 \%$ of the stent diameter at nominal pressure in air). Sample $3\left(3.5 / 12 \mathrm{~mm}\right.$ ) was expanded in a dry state (in air at $18^{\circ} \mathrm{C}$ ). Samples 4 and $5(3.5 / 23 \mathrm{~mm})$ were expanded in sterile water at $37^{\circ} \mathrm{C}$ after being immersed for half a minute in water. Of note, the current 'instruction for use document' does not suggest a minimum time of immersion in water/blood before stent implantation. In sample 5, we performed an additional oversized postdilatation at $18 \mathrm{~atm}$ with a non-compliant Quantum Maverick ${ }^{\mathrm{TM}} 5.0 / 12 \mathrm{~mm}$ balloon catheter (Boston Scientific, Natick, MA, USA). In sample 5 (which was partially postdilated with an oversized balloon), the monorail sections of both stent balloon and postdilatation balloon were wired with a single 0.014 " guidewire to allow alignment of the postdilatation balloon with a minimum manipulation. Following stent expansion, samples were very carefully placed in a sterile petri plate; any manipulation was minimised. Consecutively, DES were dried under laminar air flow at $18^{\circ} \mathrm{C}$; we did not perform any ethanol-based or vacuum-based drying of the samples.

\section{Scanning electron microscopic analysis}

A Phillips XL30 scanning electron microscope was used to perform standard SEM imaging; further options of the system (ESEM FEG in combination with EMRAM MCS-A1 of $\mu$ Candela Systems) were not used in this study. We avoided any pretreatment (e.g., gold sputtering). Samples were indirectly fixed on the table of the SEM (none of the SEM images presented in this manuscript were taken in the vicinity of that fixation). A $1 \mathrm{KeV}$-protocol was applied (average working distance $10 \mathrm{~mm}$; range $6-12 \mathrm{~mm}$ sample dependent). Samples were examined at low magnifications (14-40X) to localise areas of possible coating irregularities. This step was followed by further assessment of the irregularities at higher magnifications (801000X) to confirm and characterise the irregularities and to measurement their dimensions.

\section{Results}

The various coating irregularities that were found are described in Table 1, which also refers to illustrative examples of each individual irregularity. Table 2 gives an overview of the presence and extent of coating irregularities in the different DES samples.

Table 1. Definitions and examples of DES coating irregularities observed. DES coating irregularities Definitions and examples

Primary crack Sharp-edged coating irregularity extending from the surface deep into the coating, sometimes with exposure of underlying stent/primer (e.g., Fig. 1A-F).

Secondary crack Sharp-edged irregularity extending perpendicular to primary cracks (Fig. 4A and 4D).

Tiling Local outward displacement of coating with the formation of tile-like structures resembling the ridge of a roof (e.g., Fig. 2F).

Lifting of coating Upwards displacement of the central portion of the coating on a cross-link while still being connected with the surrounding coating (e.g., Fig. 3A)

Fragment of coating Detached piece of coating that may or may not keep minimum contact to the rest of the coating (e.g., Fig. 2C and 3D).

Total detachment Total loss of coating with exposure of of coating underlying stent/primer (e.g., Fig. 3E and 4C) 
Table 2. Presence and extent of coating irregularities in the different DES samples.

\begin{tabular}{|c|c|c|c|c|c|}
\hline & $\begin{array}{c}\text { DES sample } \\
1 \\
\text { (expanded in air } \\
\text { at } 18^{\circ} \mathrm{C} \text { at } 6 \mathrm{~atm} \\
\text { without postdilatation) }\end{array}$ & $\begin{array}{c}\text { DES sample } \\
2 \\
\text { (expanded in air } \\
\text { at } 18^{\circ} \mathrm{C} \text { at } 10 \mathrm{~atm} \\
\text { without postdilatation) }\end{array}$ & $\begin{array}{c}\text { DES sample } \\
3 \\
\text { (expanded in air } \\
\text { at } 18^{\circ} \mathrm{C} \text { at } 14 \mathrm{~atm} \\
\text { without postdilatation) }\end{array}$ & $\begin{array}{c}\text { DES sample } \\
4 \\
\text { (expanded in water } \\
\text { at } 37^{\circ} \mathrm{C} \text { at } 14 \mathrm{~atm} \\
\text { without postdilatation) }\end{array}$ & $\begin{array}{c}\text { DES sample } \\
5 \\
\text { (expanded in water } \\
\text { at } 37^{\circ} \mathrm{C} \text { at } 14 \text { atm } \\
\text { followed by oversized } \\
\text { partial postdilatation) }\end{array}$ \\
\hline Primary cracks & + & ++ & +++ & +++ & ++++ \\
\hline Secondary cracks & - & - & - & - & + \\
\hline Tiling & - & - & + & + & + \\
\hline Lifting of coating & - & - & - & + & ++ \\
\hline Fragment of coating & - & - & - & + & + \\
\hline Total detachment of coating & - & - & - & + & + \\
\hline
\end{tabular}

-: irregularity absent; + to ++++: irregularity present with increase in severity from + to ++++

\section{Cracks in DES coating}

The examination of a total of 130 SEM images of the BioMatrix DES demonstrated the presence of (primary) cracks in the coating which were located mainly on the inner curvature of stent loops (Figures 1 and 2). Depending on the degree of stent overstretch, the width of cracks measured up to $64 \mu \mathrm{m}$ and the length reached up to $74 \mu \mathrm{m}$. When examining the interior aspect of some cracks that occurred following mild overstretching of the stents, we saw sharp edges and a rough interior surface of the coating (Figure 2D). Some cracks exposed the underlying stent/primer (Figure 2D). In addition, relatively small polymer fragments (Figure $2 \mathrm{C}$ ) were noticed inside cracks and in the vicinity of cracks (visible in sample 3). There was an increase in size and number of cracks in stents expanded at

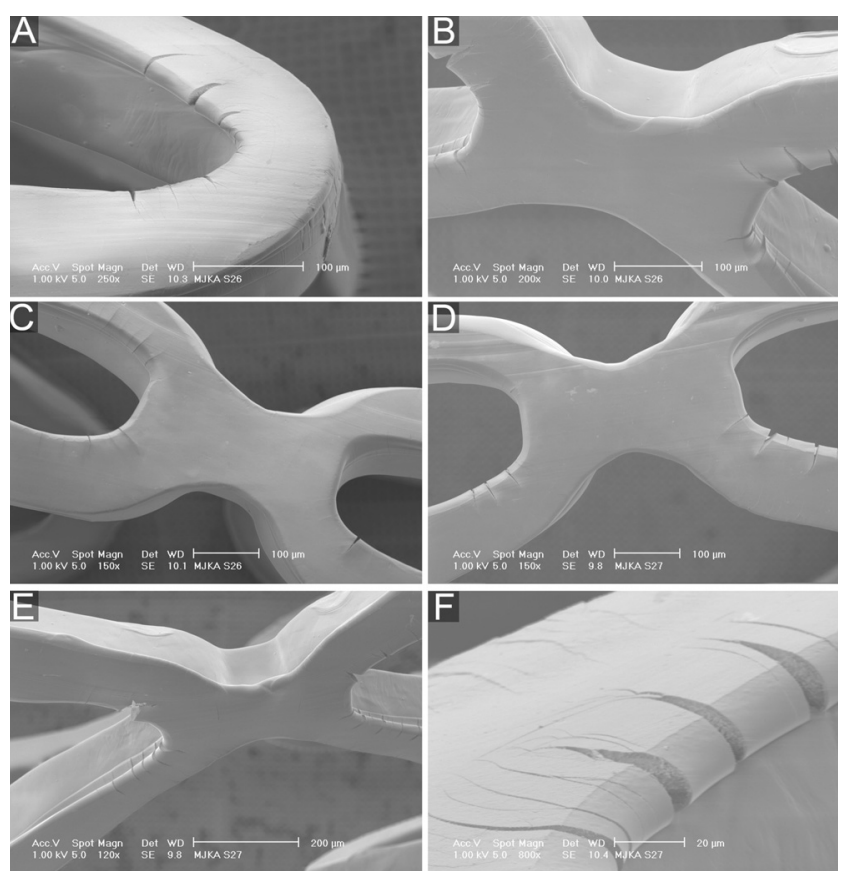

Figure 1. A,B, and C: SEM images of DES sample 1 (after 6 atm expansion in air). D,E, and F: SEM images of DES sample 2 (after 10 atm expansion in air). See results section for further details. Careful examination of panel $1 C$ and $1 D$ demonstrate an increase in size of cracks in sample 2 versus sample 1.
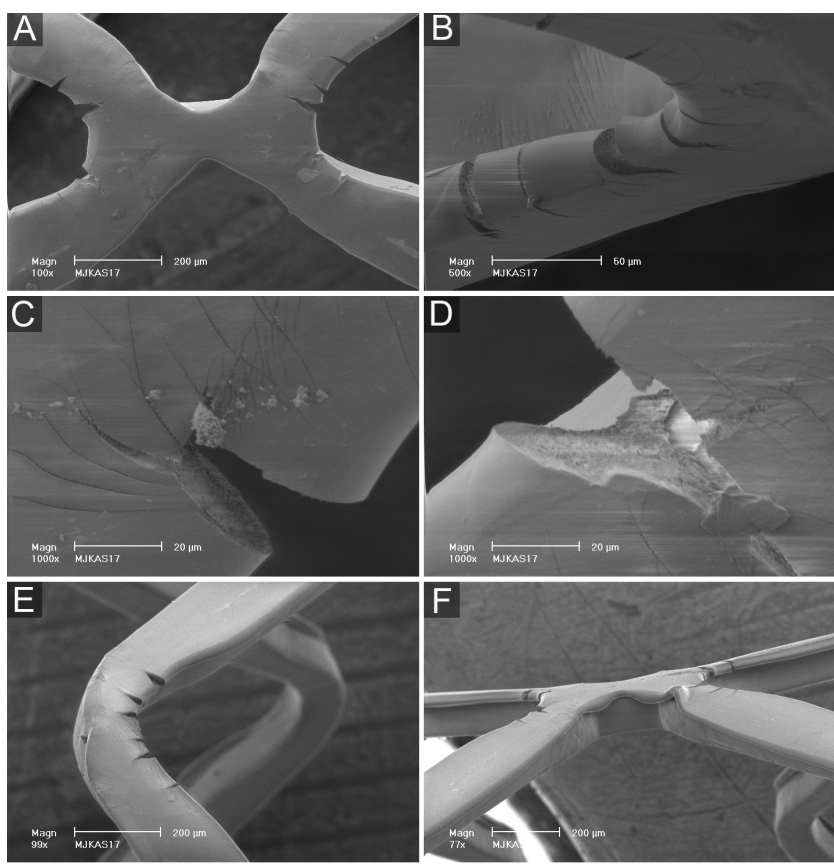

Figure 2. SEM images of DES sample 3 (expanded in air). See results section for further details.

increasing pressures (from 6 to 10 and 14 atm in air, respectively). Cracks tended to be larger in sample 4 (expanded in water) (Figure 3B-C). Postdilatation of the expanded DES with a large, oversized balloon, was associated with relatively large cracks (Figures 4B and 4C). In addition, we noticed secondary cracks that showed an orientation perpendicular to the primary cracks (sample 5; Figures 4A and 4D).

\section{Tiling and lifting of fragments of DES coating}

Following stent expansion at $14 \mathrm{~atm}$ in air, we occasionally noticed tiling of the coating at cross-links between two rings up to a maximum height of $63 \mu \mathrm{m}$ (sample 3; Figure 2F) with exposure of the stent/primer at its base. In sample 4 and sample 5 (after 14 atm in water and after partial oversized postdilatation in water, respectively), there was more tiling, lifting of portions of the polymer (in particular at the cross-links) (Figures $3 \mathrm{~A}, 3 \mathrm{E}, 4 \mathrm{E}$, and $4 \mathrm{~F}$ ) in 

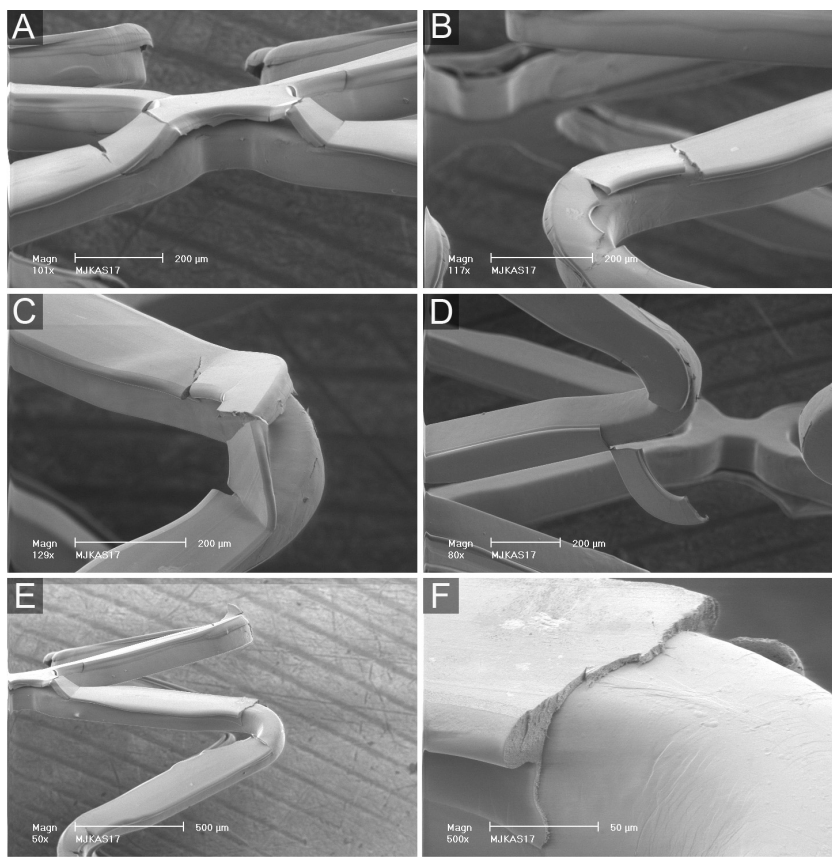

Figure 3. SEM images of DES sample 4 (expanded in water). See results section for further details.
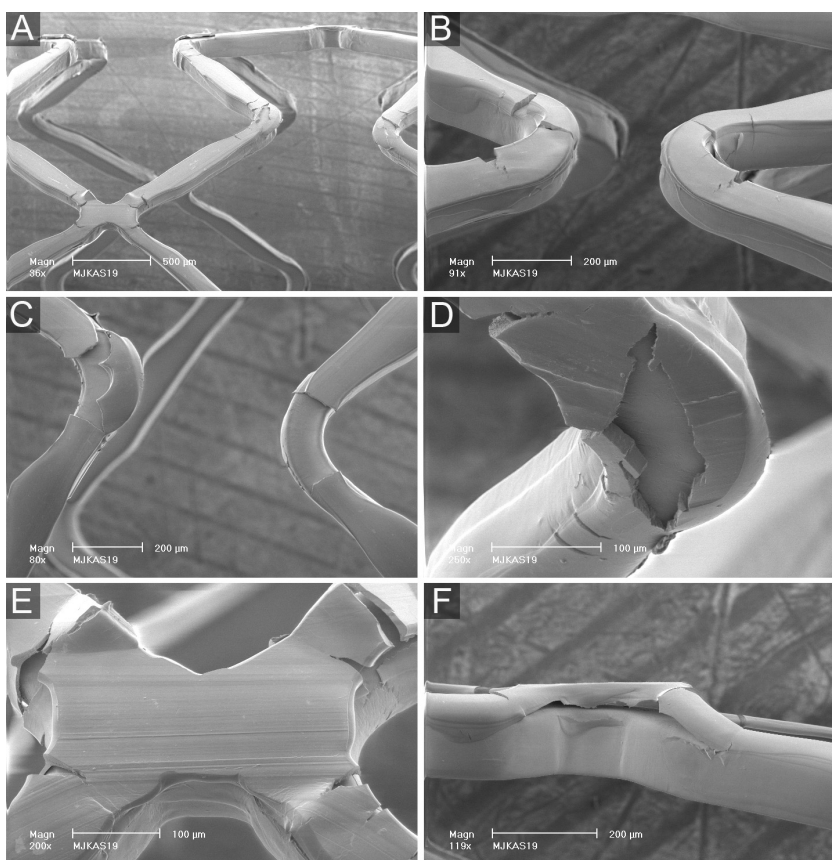

Figure 4. SEM images of DES sample 5 (after expansion in water followed by aggressive oversized partial postdilatation). See results section for further details. Of note, panel $4 F$ demonstrates the same irregularity as panel $4 E$ but shown from a lateral view.

abluminal direction (which in the physiological situation would be apposed to the vessel wall). The size of the largest lifted portion of polymer measured $93 \times 344 \mu \mathrm{m}$.

\section{Detachment of fragments of DES coating}

In DES expanded in water, several partially detached fragments of coating were detected (maximum size $85 \times 310 \mu m$; Figure 3D) while there were only few sites with total loss of coating
(Figures 3E and 3F), indicating sites of total detachment of polymer fragments (maximum size 106×350 $\mu \mathrm{m}$ ).

\section{Discussion}

The introduction of DES with biodegradable polymer coatings has the intention to reduce potential unfavourable (late) events of DES. In the LEADERS trial, the biodegradable polymer-based BioMatrix DES confirmed non-inferiority vs. the durable polymer-based Cypher stent. ${ }^{13}$ However, the PLA-based coating of the BioMatrix stent has physical properties (e.g., elasticity that is lower than in many durable polymers) that may lead to irregularities and defects of the coating following stent expansion and/or oversized partial postdilatations, as suggested by the observations of the present study.

\section{Elasticity of DES coating and irregularity formation}

The appearance of cracks in the coating (on inner curvatures of loops) and tiling of the coating (on cross-links) suggests a relatively low elasticity of this PLA coating in comparison to many durable polymers. This results in a higher susceptibility to the development of coating irregularities during more than mild overstretch of the stent. In particular, the inner curvature of loops is exposed to higher forces during unfolding of the stent, which may explain the predominance of cracks on the inner curvatures of loops which were found at nominal pressure (6 atm) and after very mild overstretch of the stent (103\% and $107 \%$ of diameter at nominal pressure) following stent expansion at $10 \mathrm{~atm}$ and $14 \mathrm{~atm}$.

In the absence of information on a minimum time of immersion in water or blood in the current instruction for use (IFU) document of the BioMatrix stent, we immersed samples 4 and 5 for 30 seconds in water to simulate the shortest feasible time required to reach and stent an easily accessible target lesion. But apparently, that brief immersion in water was not sufficient to improve the elasticity of this coating. Immersion for at least 60 seconds may have been more desirable to improve its physical properties (John Shulze, CTO Biosensors; personal communication on January 29, 2009).

In BioMatrix DES, the size of the detached polymer fragments was larger than observed in conventional durable polymer-based DES ${ }^{17}$ As shown by our experiments, postdilatation of the BioMatrix stent with an extremely oversized non-compliant balloon may result in worsening of cracks in the coating. Even further secondary cracks may be the result of such aggressive postdilatations, which may be required in clinical scenarios when stents are implanted in the transition between the left main stem and one of its major branches.

\section{Implications}

Our in vitro observations need to be cautiously interpreted, as recent clinical data suggested that the BioMatrix DES is safe and effective, ${ }^{13}$ and no correlation has been established between coating defects of this (or other) DES and the observed clinical performance in vivo in animals or humans.

As this PLA polymer is expected to be totally degraded within the generally advised 12 months of dual antiplatelet therapy following 
DES implantation, cracks and similar irregularities of the coating are unlikely to result in late unfavourable cardiac events.

The manufacturer of the BioMatrix stent recommends (in the 'instruction for use document') to generally select a stent diameter that results in no more than $10 \%$ overexpansion of the stent after implantation. However, it is a matter of fact that experienced interventional cardiologists occasionally apply much more aggressive partial overexpansions to DES during some of their procedures (e.g., $\mathrm{PCl}$ of left main bifurcation disease; major bifurcation lesions that involve considerable vessel tapering; or coronary stenoses that involve aneurismatic coronary segments). Our overstretch test in sample 5 refers to these scenarios, showing that some de-lamination of this DES could occur at sites of maximum mechanical stress if stents are overstretched in such a way.

Detachment of polymer fragments could then theoretically reduce the antiproliferative potential of DES (because of loss of the antiproliferative drug). While the clinical effect of loss in antiproliferative potential may be negligible, embolisation of substantial pieces of the coating could lead to luminal obstruction on a microvascular level that could be associated with some degree of periprocedural cardiac marker release.

In particular, embolisation of fragments of the coating is more likely if the DES is postdilated (i.e., overstretched), but partially, not well apposed against the vessel wall (e.g., when covering the ostium of a large side branch; or in the vicinity of an ex-centric, calcified stenosis). Our observations are no more than hypothesis-generating; further in vitro tests in challenging and realistic vessel phantoms of coronary lesions should be performed, which may include quantitative measurements of particles released from a stent during (and following) its expansion. ${ }^{18}$ In addition, post hoc analyses of data of the LEADERS trial may assess potential relations between periprocedural cardiac marker release and procedural details such as implantation and postdilatation pressures and degree of stent overstretch.

Nevertheless, until further data have been accumulated, interventional cardiologists may consider our findings when stenting lesions that involve major bifurcations -in particular left main bifurcations with a need for final kissing balloon inflations. In the LEADERS trial, there was only a small difference in 30-days myocardial infarction rate, favouring Cypher vs. BioMatrix but that difference did not reach statistical significance. ${ }^{13}$

\section{Limitations}

As an inherent limitation of bench-side studies, the present in vitro study does not exactly mimic the conditions in vivo. In our experimental set-up, we did not implant stents in vessels or vascular phantoms that could provide some load to stabilise the stent coating against the vessel wall. Implantation in vessels or vascular phantoms might have reduced tiling and displacement of polymer fragments. On the other hand, current simulated vessel phantoms generally do not match the physiological situation of (often) rigid and calcified atherosclerotic lesions, in which we could only speculate on how high balloon pressures or "dog-boning" as a result of partial stent overstretch (of the stent extremities) may affect the integrity of this stent's coating. ${ }^{19}$ Moreover, we avoided any additional defect that could have resulted from scratching the DES along (calcified) vessel walls or from regaining the DES out of vascular phantoms or specimens.

The design of our study is qualitative; quantification of the incidence and size of irregularities (in particular of polymer fragments) may be subject of future research. The findings of our study apply to BioMatrix stents with 9 crowns; we did not test stents with 6 crowndesign (stent diameter $<3.5 \mathrm{~mm}$ ).

We cannot fully exclude that drying this DES (following expansion in water) may have modified some coating irregularities, while stent expansion in air may have worsened its physical properties. Assessment of the post-expansion DES morphology by environmental SEM may be an option to avoid such effects.

Although much effort was taken to handle stents with maximum care, we cannot exclude that an individual defect may have been aggravated (e.g., that an almost completely detached fragment of coating could have lost its final connection). However, there are scenarios in clinical practice where such stents and their coating will be exposed to much rougher actions: if, for instance, a used noncompliant balloon catheter is (re-)advanced into the stent; oversized high-pressure postdilatation is performed; or kissing balloon dilatation is performed at a major bifurcation (the latter represents a combination of stent overstretch and shear stress applied to the coating).

\section{Conclusions}

SEM assessment suggests a relatively low elasticity of the biodegradable coating on BioMatrix stents. At nominal pressure, stents showed predominantly mild cracks of the coating, while cracks increased after slight overstretch. Aggressive overexpansion of the stent, such as sometimes required in left main bifurcation stenting, worsened cracks and led to some detachment of fragments of the coating in vitro.

\section{Acknowledgements}

We are very grateful to Jan Feijen, MSc PhD, Professor in Biomaterials and Polymer Chemistry and Scientific Director of the Institute for Biomedical Technology (BMTI) at the University of Twente in Enschede for his valuable suggestions and for proofreading our manuscript.

\section{References}

1. Colombo A, Drzewiecki J, Banning A, Grube E, Hauptmann K, Silber S, Dudek D, Fort S, Schiele F, Zmudka K, Guagliumi G, Russell ME (2003) Randomized study to assess the effectiveness of slow- and moderate-release polymer-based paclitaxel-eluting stents for coronary artery lesions. Circulation 108:788-794.

2. Moses JW, Leon MB, Popma JJ, Fitzgerald PJ, Holmes DR, O'Shaughnessy C, Caputo RP, Kereiakes DJ, Williams DO, Teirstein PS, Jaeger JL, Kuntz RE. Sirolimus-eluting stents versus standard stents in patients with stenosis in a native coronary artery. N Engl J Med 2003;349:1315-23.

3. Serruys PW, Degertekin M, Tanabe K, Abizaid A, Sousa JE, Colombo A, Guagliumi G, Wijns W, Lindeboom WK, Ligthart J, de Feyter PJ, Morice MC. Intravascular ultrasound findings in the multicenter, randomized, doubleblind RAVEL (RAndomized study with the sirolimus-eluting VElocity balloon-expandable stent in the treatment of patients with de novo native coronary artery Lesions) trial. Circulation 2002;106:798-803. 
4. Kastrati A, Mehilli J, Pache J, Kaiser C, Valgimigli M, Kelbaek H, Menichelli M, Sabate M, Suttorp MJ, Baumgart D, Seyfarth M, Pfisterer ME, Schomig A (2007) Analysis of 14 trials comparing sirolimus-eluting stents with bare-metal stents. N Engl J Med 356:1030-1039.

5. Schomig A, Dibra A, Windecker S, Mehilli J, Suarez de LJ, Kaiser C, Park SJ, Goy JJ, Lee JH, Di LE, Wu J, Juni P, Pfisterer ME, Meier B, Kastrati A. A meta-analysis of 16 randomized trials of sirolimus-eluting stents versus paclitaxel-eluting stents in patients with coronary artery disease. J Am Coll Cardiol 2007;50:1373-80.

6. Spaulding C, Daemen J, Boersma E, Cutlip DE, Serruys PW. A pooled analysis of data comparing sirolimus-eluting stents with bare-metal stents. N Engl J Med 2007;356:989-97.

7. Stettler C, Wandel S, Allemann S, Kastrati A, Morice MC, Schomig A, Pfisterer ME, Stone GW, Leon MB, de Lezo JS, Goy JJ, Park SJ, Sabate M, Suttorp MJ, Kelbaek H, Spaulding C, Menichelli M, Vermeersch P, Dirksen MT, Cervinka P, Petronio AS, Nordmann AJ, Diem P, Meier B, Zwahlen M et al. Outcomes associated with drug-eluting and bare-metal stents: a collaborative network meta-analysis. Lancet 2007;370:937-48.

8. Daemen J, Serruys PW. Drug-eluting stent update 2007: part I. A survey of current and future generation drug-eluting stents: meaningful advances or more of the same? Circulation 2007;116:316-28.

9. Ostojic M, Sagic D, Jung R, Zhang YL, Nedeljkovic M, Mangovski L, Stojkovic S, Debeljacki D, Colic M, Beleslin B, Milosavljevic B, Orlic D, Topic D, Karanovic N, Paunovic D, Christians U. The pharmacokinetics of Biolimus A9 after elution from the Nobori stent in patients with coronary artery disease: the NOBORI PK study. Catheter Cardiovasc Interv 2008;72:901-8.

10. Storger H, Grube E, Hofmann M, Schwarz F, Haase J. Clinical experiences using everolimus-eluting stents in patients with coronary artery disease. J Interv Cardiol 2004;17:387-90.

11. Vogt F, Stein A, Rettemeier G, Krott N, Hoffmann R, vom DJ, Bosserhoff AK, Michaeli W, Hanrath P, Weber C, Blindt R. Long-term assessment of a novel biodegradable paclitaxel-eluting coronary polylactide stent. Eur Heart J 2004;25:1330-40.
12. Grube E, Buellesfeld L. BioMatrix Biolimus A9-eluting coronary stent: a next-generation drug-eluting stent for coronary artery disease. Expert Rev Med Devices 2006;3:731-41.

13. Windecker S, Serruys PW, Wandel S, Buszman P, Trznadel S, Linke A, Lenk K, Ischinger T, Klauss V, Eberli F, Corti R, Wijns W, Morice MC, di Mario C, Davies S, van Geuns RJ, Eerdmans P, van Es GA, Meier B, Juni P. Biolimus-eluting stent with biodegradable polymer versus sirolimus-eluting stent with durable polymer for coronary revascularisation (LEADERS): a randomised non-inferiority trial. Lancet 2008;372: 1163-73.

14. Ormiston J, Webster M, Ruygrok P, Stewart J, Scott D, Currie E, Panther M, Shaw B. Polymer integrity after Cypher and Taxus stent implantation: A scanning electron microscope study. http://www.tctmd.com. 2005.

15. Otsuka Y, Chronos NA, Apkarian RP, Robinson KA. Scanning electron microscopic analysis of defects in polymer coatings of three commercially available stents: comparison of BiodivYsio, Taxus and Cypher stents. J Invasive Cardiol 2007;19:71-6.

16. Kitahara H, Kobayashi Y, Yamaguchi M, Fujimoto Y, Nameki M, Nakayama T, Kuroda N, Komuro I. Damage to polymer of undelivered sirolimus-eluting stents. J Invasive Cardiol 2008;20:130-3.

17. Basalus MW, Ankone MJ, van Houwelingen GK, de Man FH, von Birgelen C. Coating irregularities of durable polymer-based drug-eluting stents as assessed by scanning electron microscopy. Eurolntervention 2009;5:157-165.

18. Conti JC, Strope E, Ramesh R, Conti C, Watkins A. A Comprehensive Protocol and Procedural Considerations Designed to Evaluate the Shedding of Particles from Drug Eluting Stents. Proceedings from the Materials \& Processes for Medical Devices Conference, 2007.

19. von Birgelen C, Mintz GS, Bose D, Baumgart D, Haude M, Wieneke H, Neumann T, Brinkhoff J, Jasper M, Erbel R. Impact of moderate lesion calcium on mechanisms of coronary stenting as assessed with three-dimensional intravascular ultrasound in vivo. Am J Cardiol 2003;92:5-10. 